\title{
A new workflow on bone marrow aspiration and biopsy during the COVID-19 pandemic
}

Hui Xiao, Jing He, Sanyun Wu, Hui Shen, Li Liu, Xiaoyan Liu, Yalan Yu, Tingting Huang, Ping Luo, Fuling Zhou

Department of Haematology, Zhongnan Hospital of Wuhan University, Wuhan, China

Submitted: 21 April 2021; Accepted: 21 July 2021

Online publication: 25 July 2021

Arch Med Sci 2021; 17 (5): 1429-1432

DOI: https://doi.org/10.5114/aoms/140395

Copyright (c) 2021 Termedia \& Banach

\section{General evaluation (Figure 1)}

1. Outpatient physicians are the frontline of the defence. For patients with no or mild symptoms, making an appointment for bone marrow and biopsy examinations after the end of the pandemic is recommended. For patients who are urgently require the above examinations, and who are without any other risk factors (recent contact history (within 14 days) of COVID-19/fever, older age, history of chronic diseases, etc.) [1], strict evaluation is required. COVID-19 screening including chest computed tomography (CT) scan [2], blood routine test, and COVID-19 nucleic acid and antibody detection test should be arranged by outpatient physicians. Only patients excluded from COVID-19 infection should make a further appointment for bone marrow examinations.

2. Body temperature measurement should be performed before patients' access to the haematology laboratory. Any patients with body temperature above $37.3^{\circ} \mathrm{C}[3]$ should be escorted to the fever clinic for COVID-19 investigation. In addition to the conventional informed consent form for bone marrow aspiration and biopsy operation, a specialized consent form designed for the COVID-19 pandemic should also be signed by patients, in order to exclude relevant risk factors and tell them "things to note".

3. Bone marrow aspiration can exclusively be performed after physicians' double-check for the results from COVID-19 screening (results within 3 days) and informed consent forms have been signed.

\section{Staff management}

1. Staff preparing for work should understand the principles of hand hygiene, standard protocols for the donning and doffing of protective clothing, zone divisions, disinfection norms, and disposal of medical waste. Training courses via the Internet on nosocomial and COVID-19 infection control are provided for everyone, ensuring that each staff member understands how to protect him/herself from infection by passing a test after training.

2. All laboratory staff, including medical technicians, should report the presence of abnormal symptoms [4] such as fever, fatigue, dry cough, pharyngalgia, diarrhoea, dyspnoea, etc. on a daily basis. Anyone with any of the above risk factors should be moved to the fever clinic immediately.

\author{
Corresponding author: \\ Prof. Fuling Zhou \\ Department \\ of Haematology \\ Zhongnan Hospital \\ of Wuhan University \\ Wuhan, China \\ E-mail: \\ zhoufuling@whu.edu.cn
}




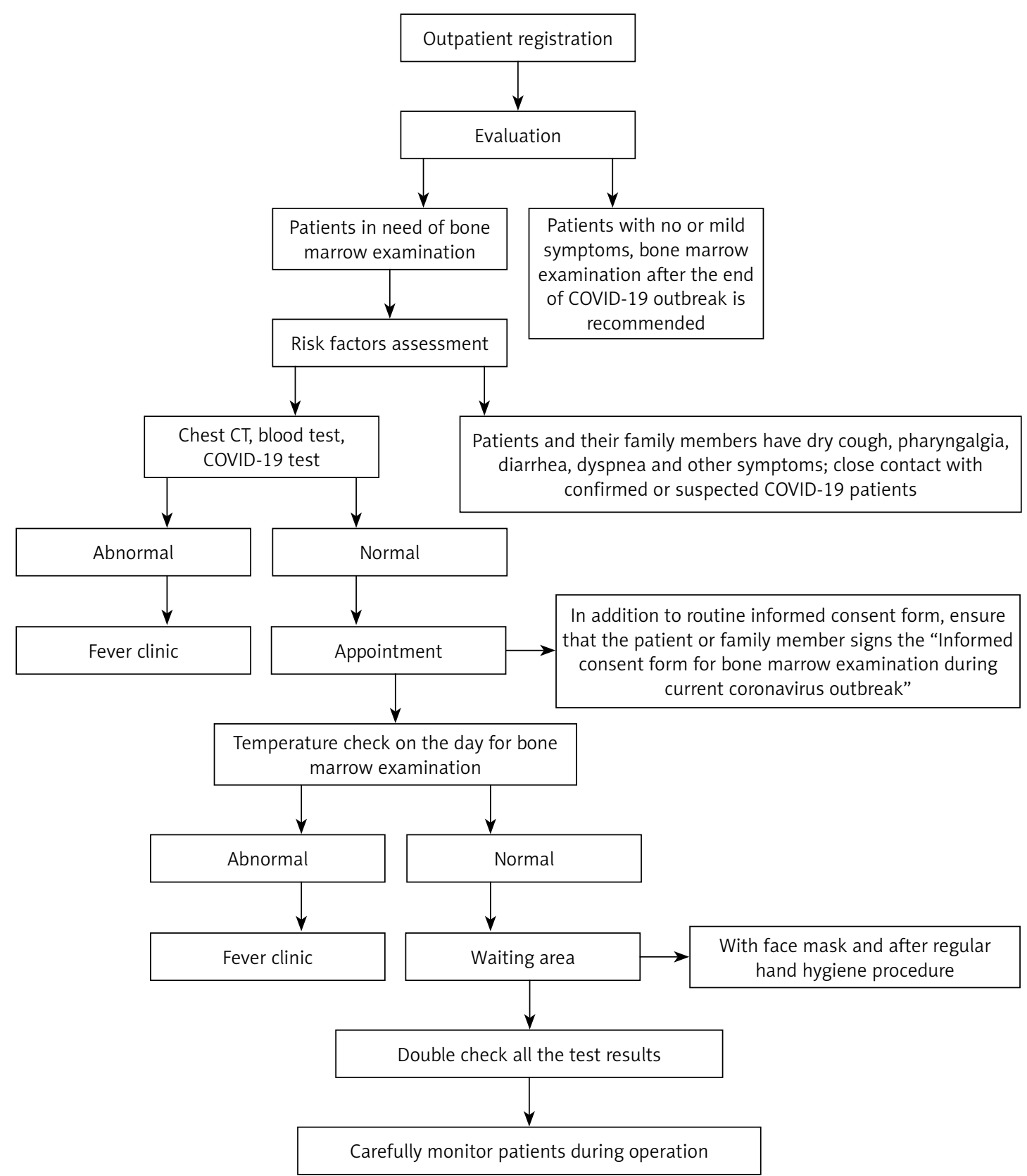

Figure 1. Evaluation steps for bone marrow aspiration and biopsy during the pandemic of COVID-19

3. Hand hygiene should be strictly implemented according to the "six-step hand washing technique" [5]. Another option would be the application of quick-drying hand disinfectant for 2 min, but this cannot replace the "six-step hand washing technique".

4. The staff, especially physicians and medical technicians who are in direct contact with patients, must be dressed in overalls as un underlayer with disposable waterproof isolation clothing/protective clothing on top, double latex gloves, N95 masks, goggles/protective face screen, and disposable head and shoe covers. Any of the items above should be replaced immediately if they become contaminated.
5. Food and drink are strictly forbidden in the working area.

6. Staff should be isolated at home during breaks; going out or attending gatherings should be minimized.

\section{Implementation of the updated standard operating procedure}

1. Operating materials. Disposable kits for bone marrow examination must be used for all patients.

2. Patient position. It is preferable to choose the posterior superior iliac spine as the site of operation, and it is better for the patient in the 
prone or lateral position instead of in the supine position (to minimize the chance of faceto-face contact).

3. Operator preparation. The operator should have second-level protection, and an assistant should be by his/her side.

\section{Management of patients and assistants}

1. Examinations can only be implemented by appointment during the COVID-19 pandemic.

2. Patients for bone marrow examination should provide accurate personal information and results from COVID-19 screening.

3. Patients and their assistants in the waiting area of the laboratory must routinely wear masks [6], sterilize their hands, and measure their body temperature.

4. Patients and their assistants should go through a restricted lane; a "one-on-one" mode is preferable to patients and their assistants, in principle.

\section{Disinfection of the working environment}

1. Air disinfection: keeping windows open to improve ventilation in wards is advisable - half an hour each time, twice a day. An air sterilizer can also be used as another option $-2 \mathrm{~h}$ each time, 4 times a day. Use of a central air-conditioning system is prohibited.

2. Cleaning and disinfection of object surfaces: according to the conventional disinfection method, chlorine-based disinfectant can be applied with an effective chlorine concentration $1000 \mathrm{mg} / \mathrm{l}$ (spraying is prohibited), and disinfectant can be wiped away with fresh water after $30 \mathrm{~min}$ - at least twice a day.

3. Floor disinfection: chlorine-based disinfectant with a concentration of $2000 \mathrm{mg} / \mathrm{l}$ should be used to mop the floor; the disinfectant should be rinsed off with fresh water after $30 \mathrm{~min}$ - at least twice a day.

\section{Disinfection for medical equipment}

1. $75 \%$ ethanol [7] or $1000 \mathrm{mg} / \mathrm{l}$ chlorine-based disinfectant can be adopted if there is no blood contamination on the surface of the equipment after treatment (spraying is prohibited).

2. If the equipment is contaminated by blood or secretions from COVID-19-infected patients (or susceptible patients), the contaminated surface should be cleaned with 2000-5000 mg/l chlorine-based disinfectant, left for $30 \mathrm{~min}$, and then wiped off with $75 \%$ alcohol under proper protective conditions.

\section{Medical waste disposal}

All the waste generated from patients should be securely contained with double yellow clinical waste bags [3], separately labelled, and dealt with using the hospital's standard process for infectious waste.

\section{Other matters to note}

1. Hospitals are particularly hazardous areas; haematology patients should be lectured on self-protection due to their low immunity.

2. Highly visible signs should be posted to warn of the dangers posed by moving around the hospital.

3. A higher frequency of disinfection in the working environment and for medical equipment is strongly advised after performing operations on COVID-19-positive patients.

\section{Limitation of the workflow}

We are still working on the evolution of this workflow due to the gradual discovery of its limitations. For instance, what should haematologists do when encountering COVID-19+ ${ }^{+}$patients who are uncooperative (e.g. infants, patients with mental disorders, etc.)? Although we lack experience in facing such a special community, we are confident that more flexible recommendations will be incorporated into the workflow in the near future.

\section{Summary}

Established from the notion that fighting a pandemic is a comprehensive action requiring efforts from all sides, the workflow consists not only of actions that should be undertaken by physicians, but also those that should be performed by technicians, patients, and their assistants. Despite the fact that the effectiveness and safety of the workflow have been proven in our daily work, we still strongly suggest that indications of bone marrow aspiration and biopsy should be strictly verified to avoid nosocomial infection under the current pandemic of COVID-19. To reduce the odds of infection, senior haematologists should evaluate the necessity of any operation before its implementation and screen the risk factors for the patients.

\section{Acknowledgments}

Hui Xiao and Jing He contributed equally to this work.

\section{Conflict of interest}

The authors declare no conflict of interest.

\section{References}

1. Zhou F, Yu T, Du R, et al. Clinical course and risk factors for mortality of adult inpatients with COVID-19 in Wuhan, China: a retrospective cohort study. Lancet 2020; 395: 1054-62. 
2. Zhou L, Liu HG. Early detection and disease assessment of patients with novel coronavirus pneumonia. Zhonghua Jie He He Hu Xi Za Zhi 2020; 43: 167-70.

3. Ying HJ, Lin C, Zhen SC, et al. A rapid advice guideline for the diagnosis and treatment of 2019 novel coronavirus (2019-nCoV) infected pneumonia (standard version). Mil Med Res 2020; 7: 4

4. Guan WJ, Ni ZY, Hu Y, et al. Clinical characteristics of coronavirus disease 2019 in China. N Engl J Med 2020; 382: 1708-20.

5. World Health Organization. WHO guidelines on hand hygiene in health care. World Health Organization, Geneva 2009.

6. Agolini G, Raitano A, Viotti PL, Vitali M, Zorzut F. SARS: diagnostica, terapia e soprattutto prevenzione [SARS: diagnosis, therapy, and especially prevention]. Ann Ig 2004; 16: 211-24.

7. Kampf G, Todt D, Pfaender S, Steinmann E. Persistence of coronaviruses on inanimate surfaces and their inactivation with biocidal agents. J Hosp Infect 2020; 104 246-51. 